MIR-382-5P TARGETING IL-33 GENE AS BIOMARKER TO PREDICT SUBCLINICAL ATHEROSCLEROSIS PROGRESSION IN PATIENTS WITH EARLY RHEUMATOID ARTHRITIS

T.H. Cheng ${ }^{1}$, Q. Shang ${ }^{1}$, E. K. M. Li ${ }^{1}$, M. Li ${ }^{1}$, W.Y. Mak ${ }^{1}$, K.Y. Kwok ${ }^{2}$, I.C.W. Yim ${ }^{3}$ P.C.H. Wong ${ }^{1}$, V.W.N. Lao ${ }^{4}$, S.H.T. Pang ${ }^{4}$, E.W.L. Kun ${ }^{5}$, L.S. Tam ${ }^{1} .{ }^{1}$ Department of Medicine and Therapeutics, The Chinese University of Hong Kong; ${ }^{2}$ Department of Medicine, Queen Elizabeth Hospital; ${ }^{3}$ Department of Medicine, Tseung Kwan O Hospital, ${ }^{4}$ Department of Medicine, Kwong Wah Hospital, ${ }^{5}$ Department of Medicine, Tai Po Hospital, Hong Kong, Hong Kong

Background: Patients with rheumatoid arthritis(RA) had increased risk of cardiovascular disease(CVD). IL-33, a member of the IL-1 family, plays an important role in the pathogenesis of RA and development of CVD. Yet, plasma IL-33 level was not detectable in most subjects which limits it's utility as a biomarker for CVD. Meanwhile, microRNAs(miRNAs) targeting IL-33 gene expression might play a role.

Objectives: To ascertain if dysregulated miRNAs targeting IL-33 gene expression in earlyRA patients were associated with subclinical atherosclerosis progression

Methods: 73 ERA patients were recruited for this 1 year cohort study. Potential miRNAs binding to $I L-33$ gene were predicted by miRanda. 10 miRNAs with the highest possibility of targeting functional sites of $I L-33$ gene were quantified in cell free plasma samples. cel-miR-39 was used as spike-in control. Carotid plaque (CP) was identified using high-resolution ultrasound annually. Plaque progression (PP) was defined as an increased region harbouring plaque.

Results: CPs were identified in $25(34 \%)$ and $31(43 \%)$ subjects at baseline and month 12 respectively. 16 (22\%) subject had plaque progression(PP +group). At baseline, subjects in PP + group were older, with lower pain and patient global scores, a higher proportion on conventional synthetic DMARDs, and higher cardiovascular risk compared to patients without plaque progression (PP-) (table 1) Plasma level of miR-382-5 $p$ in the PP + group was significantly higher than that in the PP- group after adjusting for baseline difference (table 1). Using multivariate logistic regression, miRNA-382-5 $\mathrm{p}$ was an independent predictor for plaque progression(OR:2.534, 95\% $\mathrm{Cl}=1.079-5.952, \mathrm{p}=0.033$ ) after adjustment of baseline characteristics. [AUC:0.66,95\% Cl:0.51-0.81, $\mathrm{p}=0.048$ ]. Other independent predictor included higher baseline Framingham risk score, diastolic BP and lower pain score.

Abstract THU0179 - Table 1. Characteristics of patients with and without plaque progression

\begin{tabular}{lcccc}
\hline & $\begin{array}{c}\text { No plaque progression } \\
(\mathrm{n}=57)\end{array}$ & $\begin{array}{c}\text { Plaque progression } \\
(\mathrm{n}=16)\end{array}$ & $p$ & $p^{*}$ \\
\hline Age & $50 \pm 12$ & $56 \pm 7$ & 0.011 & \\
Gender, male & $11(19.3 \%)$ & $6(37.5 \%)$ & 0.128 & \\
Franmingham Risk & $6.1 \pm 7.9$ & $14.2 \pm 11.6$ & 0.017 & \\
Score & & & & \\
log miRNA & & & & \\
miR_9_5p & $-1.14 \pm 1.48$ & $-1.23 \pm 0.97$ & 0.825 & 0.947 \\
miR_382_5 $p$ & $0.79 \pm 0.66$ & $1.38 \pm 1.29$ & 0.094 & 0.011 \\
miR_377_3p & $0.75 \pm 0.65$ & $0.96 \pm 0.85$ & 0.285 & 0.783 \\
miR_590_3p & $0.2 \pm 0.46$ & $0.13 \pm 0.16$ & 0.517 & 0.472 \\
miR_499a_5 & $-1.65 \pm 0.67$ & $-1.48 \pm 0.88$ & 0.418 & 0.370 \\
miR_145_5 $p$ & $2.43 \pm 1.35$ & $3.26 \pm 2.08$ & 0.060 & 0.169 \\
miR_542_3p & $-1.95 \pm 0.55$ & $-1.85 \pm 0.99$ & 0.615 & 0.750 \\
miR_186_5 $p$ & $2.67 \pm 1.42$ & $2.77 \pm 1.69$ & 0.811 & 0.556 \\
miR_214_3p & $-0.68 \pm 1.29$ & $-0.8 \pm 1.02$ & 0.730 & 0.849 \\
miR_496 & $-1.65 \pm 0.9$ & $-1.68 \pm 0.83$ & 0.917 & 0.538 \\
\hline
\end{tabular}

*adjusted for age, sex, baseline NRS pain, patient global score, wrist to hip ratio, plasma LDL level and Frimingham risk score

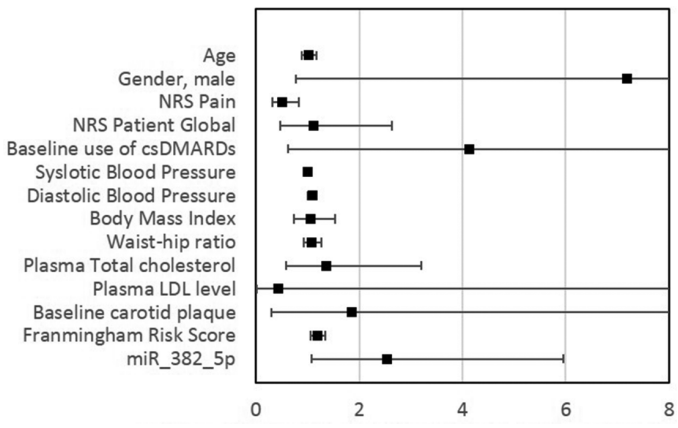

Abstract THU0179 - Figure 1. Multivariate regression analysis for plaque progression
Conclusions: miR-382-5 p was an independent predictor for progression of subclinical atherosclerosis and may serve as a novel biomarker for cardiovascular risk assessment in ERA patients.

Acknowledgements: Acknowledgement to Hong Kong Society of Rheumatology Project Fund for supporting this project.

Disclosure of Interest: None declared

DOI: 10.1136/annrheumdis-2018-eular.2395

\section{THU0180 \\ SHOULD WE INCLUDE ULTRASOUND IN THE 1987 ACR AND 2010 ACR/EULAR CLASSIFICATION CRITERIA FOR RHEUMATOID ARTHRITIS?}

W. Hamdi ${ }^{1}$, M. Sellami ${ }^{1}$, H. Riahi ${ }^{2}$, K. Maatallah ${ }^{1}$, D. Kaffel ${ }^{1}$, M. Chelli-Bouaziz ${ }^{2}$, M. F. Ladeb ${ }^{2}$, M.M. Kchir ${ }^{1} .{ }^{1}$ Rheumathology, ${ }^{2}$ Radiology, Kassab Orthopedics Institute, Manouba, Tunisia

Background: Ultrasound imaging (US) is actually considered as a crucial element of the diagnostic process in rheumatic diseases and could be the method of choice in imaging peripheral synovitis, typical feature during rheumatoid arthritis (RA). The 2010 American College of Rheumatology/European League Against Rheumatism (ACR/EULAR) classification criteria focused on early-stage RA, and were designed to remedy the deficiencies in the 1987 ACR criteria, but still lacking sensitivity.

Objectives: We undertook this study to investigate whether including the ultrasonographic data in calculating the 1987 ACR and 2010 ACR/EULAR criteria is useful in diagnosing RA.

Methods: We performed a cross-sectional study of one hundred patients with inflammatory joint pain or synovitis for more than 6 weeks and less than 2 years. An experienced radiologist performed the MSUS scan of 22 joints (2 wrists, 10 metacarpophalangeal joints MCP and 10 proximal interphalangeal joints PIP unaware of clinical and biological findings. After US assessment, patients were classified as having RA according to ACR/ EULAR 2010 criteria using clinical, biologic and radiographic data (clinical ACR87 or clinical ACR/EULAR2010). Then we calculate those criteria using US data: US-ACR87 including erosion detected in US as a criterion and US-ACR/EULAR2010 including the number of synovitis detected in US as a criterion.

Results: One hundred RA patients were included ( 77 women and 23 men) with a mean age of 51.8 years. ${ }^{16-77}$ The mean disease duration was $10.96 .{ }^{224}$ Rheumatoid factor and antibodies against cyclic citrullinated peptides were positive in $53 \%$ and $25 \%$ cases respectively. Sixty-five patients $(65 \%)$ accomplished the clinical ACR87 criteria and 55 patients (55\%) fulfilled the clinical ACR/EULAR2010 criteria for the diagnosis of RA. Using US data eighty patients $(80 \%)$ accomplished the US-ACR 87 criteria and 68 patients (68\%) fulfilled the US-ACR/EULAR2010 criteria for the diagnosis of RA. A good correlation was found between clinical and US ACR/EULAR criteria $(k=0,684, p=0,000)$. US score showed a very good sensitivity of $100 \%$, specificity of $67,5 \%$, positive predictive value of $76,9 \%$ and negative predictive value of $100 \%$ compared to clinical score. In table 1 we summarise correlation between clinical and US ACR/EULAR criteria.

Abstract THU0180 - Table 1. Correlation between clinical and US ACR/EULAR criteria

\begin{tabular}{llccc}
\hline & & \multicolumn{3}{c}{ Clinical ACR/EULAR2010 } \\
\hline US-ACR/EULAR2010 & & Yes & No & Total \\
& Yes & 50 & 18 & 68 \\
& No & 5 & 27 & 32 \\
& Total & 55 & 45 & 100 \\
\hline
\end{tabular}

Conclusions: Our study showed that including US data in the classification criteria of RA, will improve diagnostic impact of those criteria during RA management. Disclosure of Interest: None declared DOI: 10.1136/annrheumdis-2018-eular.5523

\section{THU0181 GLUCOCORTICOID USE IS AN INDEPENDENT RISK FACTOR FOR SARCOPENIA IN PATIENTS WITH RHEUMATOID ARTHRITIS - FROM THE CHIKARA STUDY -}

Y. Yamada ${ }^{1}$, M. Tada $^{2}$, K. Mandai ${ }^{3}$, N. Hidaka ${ }^{2}$, K. Inui ${ }^{1}$, H. Nakamura ${ }^{1}$. ${ }^{1}$ Department of orthopedics surgery, OSAKA CITY UNIVERSITY; ${ }^{2}$ Department of orthopedics surgery, Osaka City General Hospital; ${ }^{3}$ Department of orthopedics surgery, Osaka Social Care Center Hospital, Osaka City, Japan

Background: Patients with rheumatoid arthritis $(\mathrm{RA})$ are at higher risk of sarcopenia due to joint dysfunction and chronic inflammation. The prospective observational CHIKARA study (Correlation research of sarcopenia, skeletal muscle and disease activity in rheumatoid arthritis; registration number UMIN000023744) was 
started in 2016 to clarify the correlation between RA disease activity and sarcopenia.

Objectives: We investigated risk factors for developing sarcopenia in patients with RA.

Methods: We analysed baseline and 1 year data from the CHIKARA study. The body composition (body weight, muscle mass, fat mass, predicted bone mass, etc.) of 100 patients ( $78 \%$ women; mean age, 68 years) enrolled in this study was examined using a body composition analyzer (MC-780A; TANITA, Tokyo, Japan). Grip strength and walking speed were also measured. Laboratory data, disease activity, Health Assessment Questionnaire (HAQ) and treatment were investigated. Sarcopenia was diagnosed using the criteria of the Asia Working Group on Sarcopenia. Patients with sarcopenia onset at 1 year were detected and their characteristics were analysed. Predictors for development of sarcopenia were also investigated by uni- and multivariate analyses.

Results: Nine patients developed sarcopenia during 1 year. Glucocorticoid (GC) use was significantly more frequent among patients with sarcopenia onset $(55.6 \%)$ than among those without sarcopenia onset $(22.1 \%, p=0.029)$. Univariate analysis revealed that $\mathrm{GC}$ dosage $(r=0.217, \mathrm{p}=0.035)$, body fat mass at baseline $(r=-0.211, p=0.040)$ and change in CRP at 1 year $(r=-0.205, p=0.046)$ were significantly associated with sarcopenia onset. GC use $>2 \mathrm{mg} /$ day (Odds ratio (OR) $8.0,95 \%$ confidence interval $(\mathrm{Cl}) 1.2-54.8, \mathrm{p}=0.034)$ and body fat mass (OR 0.78 , $95 \% \mathrm{Cl} 0.61-0.98, \mathrm{p}=0.037$ ) were detected as significant factors by multivariate analysis. (table 1)

Abstract THU0181 - Table 1. Risk factors for developing sarcopenia in patients with rheumatoid arthritis

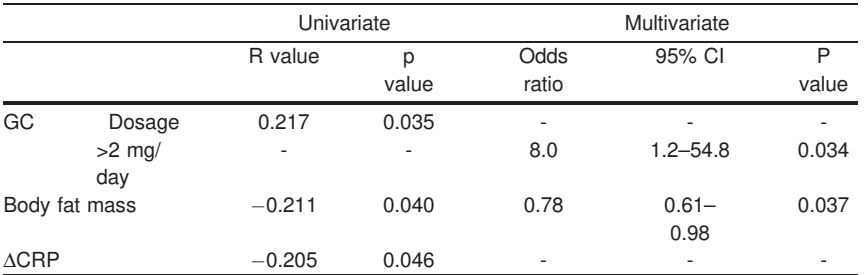

GC: glucocorticoids, $\mathrm{Cl}$ : confidence interval, $\Delta$ : change from baseline to 1 year Conclusions: RA patients using GC at $>2 \mathrm{mg} /$ day or with low fat mass were more likely to develop sarcopenia.

\section{REFERENCES:}

[1] Inui K., Koike T, Tada M, et al. Sarcopenia is apparent in patients with rheumatoid arthritis, especially those with biologics -TOMORROW study-. EULAR 2015 abstract (AB0359).

[2] Chen LK, Liu LK, Assantachai P, et al. Sarcopenia in Asia: consensus report of the Asian Working Group for Sarcopenia. J Am Med Dir Assoc 2014;15:95-101.

Disclosure of Interest: None declared

DOI: 10.1136/annrheumdis-2018-eular.1860

\section{THURSDAY, 14 JUNE 2018}

\section{Rheumatoid arthritis - biological DMARDs}

\section{THU0182 A COMPARATIVE CLINICAL STUDY OF PF-06410293, A CANDIDATE ADALIMUMAB BIOSIMILAR, AND REFERENCE ADALIMUMAB FOR THE TREATMENT OF ACTIVE RHEUMATOID ARTHRITIS}

R. Alten ${ }^{1}$, R.M. Fleischmann ${ }^{2}$, M. Pileckyte ${ }^{3}$, S.Y. Hua ${ }^{4}$, C. Cronenberger ${ }^{5}$, A. E. Bock ${ }^{6}$, K. L. Sewell ${ }^{6}{ }^{1}$ Schlosspark-Klinik, University Medicine, Berlin, Germany; ${ }^{2}$ University of Texas, Southwestern Medical Center, Metroplex Clinical Research Center, Dallas, USA; ${ }^{3}$ Lithuanian University of Health Sciences, Kaunas, Lithuania; ${ }^{4}$ Pfizer Inc, San Diego; ${ }^{5}$ Pfizer Inc, Collegeville; ${ }^{6}$ Pfizer Inc, Cambridge, USA

Background: To confirm the efficacy, safety and immunogenicity of biosimilars, a comparative clinical study is typically required.
Objectives: This double-blind, randomised, 78 week (wk) study compared the efficacy, safety and immunogenicity of PF-06410293, a candidate adalimumab biosimilar, with reference adalimumab sourced from the EU (ADA-EU), in biologic-naïve patients (pts) with active rheumatoid arthritis (RA) despite methotrexate (MTX; 10-25 mg/wk).

Methods: Pts with active RA $(n=597)$ were stratified by region and randomised (1:1) to PF-06410293 or ADA-EU (40 mg subcutaneous injection every 2 wks), with continued MTX. The primary endpoint was American College of Rheumatology $20 \%$ improvement (ACR20) at Wk 12. Therapeutic equivalence was concluded if the 2-sided $95 \%$ confidence interval $(\mathrm{Cl})$ for the difference in Wk 12 ACR20 between arms was within the symmetric equivalence margin of $\pm 14 \%$. Additionally, a $2-$ sided $90 \% \mathrm{Cl}$ was requested by the US Food and Drug Adminis tration, using the asymmetric equivalence margin of $-12 \%$ to $+15 \%$. Secondary efficacy endpoints to Wk 26 included ACR20/50/70, change from baseline Dis ease Activity Score in 28 joints [DAS28(CRP)], European League Against Rheumatism (EULAR) response, achievement of DAS28(CRP) $<2.6$, and ACR/EULAR remission. QuantiFERON-TB testing was performed at Screening and Wk 26.

Results: Pts ( $78.7 \%$ female, $81.6 \%$ seropositive) had a mean age of 52.5 years, and mean RA duration of 6.8 years. Mean baseline DAS28(CRP) was 5.9 (PF06410293) and 6.1 (ADA-EU). The observed Wk 12 ACR20 was 68.7\% (PF06410293) and $72.7 \%$ (ADA-EU) in the intent-to-treat population (figure 1). Using non-responder imputation $(\mathrm{n}=19 ; 3.2 \%)$, the treatment difference in Wk 12 ACR20 was $-2.98 \%$, and the corresponding Cls [95\% Cl $(-10.38 \%,+4.44 \%) ; 90 \% \mathrm{Cl}$ $(-9.25 \%,+3.28 \%)]$ were entirely contained within both equivalence margins (symmetric and asymmetric). The ACR20 difference ranged from $-3.98 \%$ to $+5.50 \%$ (Wks 2-26). Mean DAS28(CRP) change from baseline at Wk 26 was -2.7 and -2.8 in the PF-06410293 and ADA-EU arms, respectively. ACR50/70, EULAR response, DAS28(CRP) $<2.6$ and ACR/EULAR remission were similar between arms at each visit. Incidence of treatment-emergent adverse events (AEs) was $48.1 \%$ vs $47.8 \%$, serious AEs were $4.0 \%$ vs $4.3 \%$ (with a fatal myocardial infarc tion in the ADA-EU arm) and serious infections were $0.7 \%$ vs $1.3 \%$ for $P F-$ 06410293 and ADA-EU, respectively. Injection site reactions occurred at $1.7 \%$ vs $2.0 \%$, hypersensitivity events at $4.4 \%$ vs $8.4 \%$, pneumonia at $0.7 \%$ vs $2.0 \%$, and latent tuberculosis (based on specialist consultation for Wk 26 QuantiFERON-TB + ) at $1.7 \%$ vs $0.3 \%$ for PF-06410293 and ADA-EU, respectively. Post-dose antidrug antibody rates to Wk 26 were 44.4\% (PF-06410293) and 50.5\% (ADA-EU).

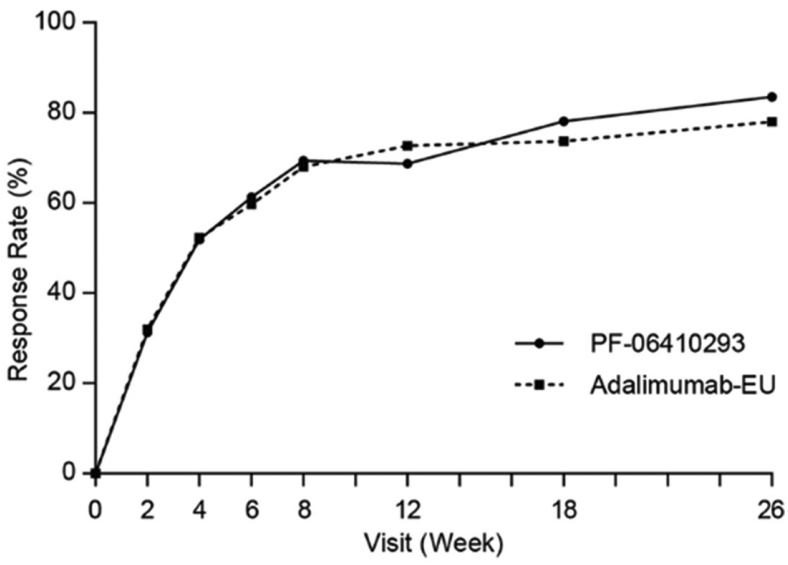

ACR=American College of Rheumatology; ITT=Intent-to-treat.

Figure 1. ACR20 response rates (ITT population)

Conclusions: The efficacy, safety and immunogenicity of PF-06410293 and ADA-EU were similar up to Wk 26 in pts with active RA on MTX. At Wk 26, pts on ADA-EU were blindly re-randomised (1:1) to continue ADA-EU or transition to PF06410293 for ongoing treatment in the study.

Disclosure of Interest: R. Alten Speakers bureau: Pfizer, R. Fleischmann Grant/research support from: Pfizer and AbbVie, Consultant for: Pfizer and AbbVie, M. Pileckyte: None declared, S. Hua Shareholder of: Pfizer, C. Cronenberger Shareholder of: Pfizer, Employee of: Pfizer, A. Bock Shareholder of: Pfizer, Employee of: Pfizer, K. Sewell Shareholder of: Pfizer, Employee of: Pfizer DOI: 10.1136/annrheumdis-2018-eular.1359 